\title{
Functional analysis of the TMPRSS2:ERG fusion gene in cisplatin-induced cell death
}

\author{
JUNQI WU ${ }^{1 *}$, LINFENG $\mathrm{CHI}^{2,3^{*}}$, ZHANGHUI CHEN ${ }^{2,4}$, XIANGHONG LU $^{5}$, SUPING XIAO ${ }^{2}$, \\ GUANGLIN ZHANG $^{2}$, JINDAN LUO ${ }^{2}$, GE-MING CHEN $^{2}$ and $\mathrm{JUN} \mathrm{YANG}^{2,6}$ \\ ${ }^{1}$ Clinical Laboratory, Jinhua Hospital of Zhejiang University, Jinhua, Zhejiang 321000; \\ ${ }^{2}$ Collaborative Innovation Center for Diagnosis and Treatment of Infectious Diseases, The First Affiliated Hospital, \\ Zhejiang University; ${ }^{3}$ The Clinical Laboratory Center, The Affiliated Stomatology Hospital, \\ Zhejiang University School of Medicine, Hangzhou, Zhejiang 310003; ${ }^{4}$ Center for Reproductive Medicine, \\ Zhejiang Academy of Medical Science, Hangzhou, Zhejiang 310013; ${ }^{5}$ Department of Pharmacy, \\ Lishui People's Hospital, Lishui, Zhejiang 323000; ${ }^{6}$ Department of Public Health, \\ Hangzhou Normal University School of Medicine, Hangzhou, \\ Zhejiang 310031, P.R. China
}

Received March 15, 2015; Accepted January 20, 2016

DOI: $10.3892 / \mathrm{mmr} .2016 .4898$

\begin{abstract}
The TMPRSS2:E-twenty-six (ETS) gene fusion occurs frequently in a high proportion of patients with prostate cancer (PCa) in Western countries, and the aberrant expression of TMPRSS2: v-ETS avian erythroblastosis virus E26 oncogene homolog (ERG), the most common form of the corresponding protein, can regulate cell migration and contribute to tumor invasion and metastasis. However, its association with other cellular events, and in particular, cell death, remain unknown. To examine the function of such fusion genes, an expression plasmid containing the TMPRSS2:ERG (T1/E5) sequence ( $\triangle \mathrm{ERG})$ from a patient sample was constructed and transiently transfected into DU145 cells, which do not express the fusion gene. It was found that the overexpression of $\triangle E R G$ significantly inhibited the ability of cisplatin to induce apoptosis in DU145 cells. By contrast, $\mathrm{VCaP}$ cells, which do contain TMPRSS2:ERG, were sensitized to cisplatin-induced apoptosis through siRNA inhibition of the fusion gene. To elucidate the underlying
\end{abstract}

Correspondence to: Dr Ge-Ming Chen, Collaborative Innovation Center for Diagnosis and Treatment of Infectious Diseases, The First Affiliated Hospital, Zhejiang University, 79 Qingchun Road, Hangzhou, Zhejiang 310003, P.R. China

E-mail:cgmcjs@msn.com

Dr Jun Yang, Department of Public Health, Hangzhou Normal University School of Medicine, 16 Xuelin Street, Hangzhou, Zhejiang 310031, P.R. China

E-mail: gastate@zju.edu.cn

*Contributed equally

Key words: fusion gene, prostate cancer, ERG, TMPRSS2, apoptosis mechanism, a stable cell line expressing the $\triangle E R G$ gene was constructed. Expression of $\triangle E R G$ did not affect cell migration, but did protect cells from DNA damage and apoptosis induced by cisplatin. Furthermore, knockdown of $\triangle E R G$ by short interfering RNA resulted in cells regaining their sensitivity to cisplatin. Finally, the gene coding for activating transcription factor 5, which is important for cell survival, may be upregulated by $\triangle E R G$. Taken together, these data point to a new function of the TMPRSS2:ERG fusion gene in regulating the apoptotic pathway.

\section{Introduction}

Prostate cancer (PCa) is the most common male malignancy, and one of the leading causes of cancer-associated mortality among men in Western countries (1). Extensive studies have been conducted in an effort to understand the genetic mechanisms underlying PCa initiation and progression. Using a systems biology approach, Tomlins et al (2) reported that the androgen response gene transmembrane protease, serine 2 (TMPRSS2) was fused to the E-twenty-six (ETS) family gene v-ETS avian erythroblastosis virus E26 oncogene homolog (ERG) and ETS variant 1 (ETV1) in certain patients with PCa. Among the various types of fusion genes, the TMPRSS2:ERG fusion is the most common, and has been identified in $40-70 \%$ of samples from patients with PCa (3).

Functional studies of TMPRSS2:ERG have suggested that it is involved in cellular proliferation, differentiation and invasion (4-7), while its association with additional cellular functions, and in particular, apoptosis, remains unknown. Shao et al (8) reported that celastrol, a nuclear factor- $\kappa \mathrm{B}$ inhibitor, can suppress TMPRSS2:ERG-expressing PCa cell growth by inducing apoptosis, implying a possible anti-apoptotic function for the TMPRSS2:ERG fusion gene.

As an effective anticancer drug, cisplatin is widely used in the treatment of many types of solid tumors, including ovarian, 
testicular, bladder, cervical, head and neck, and small-cell lung cancer $(9,10)$. Cisplatin is able to interact with DNA, and results in the formation of interstrand and/or intrastrand crosslinks (11), with such severe DNA damage considered a major factor in triggering cancer cell apoptosis. However, cisplatin treatment of PCa has been a subject of debate, as while earlier studies suggested a lack of activity, more recent studies using palliation and prostate specific antigen (PSA) endpoints have suggested a greater degree of clinical benefit (12). Therefore, it is of interest to understand why PCa cells have a low response to cisplatin-induced apoptosis, and whether the TMPRSS2:ERG fusion gene may serve a role in this response.

In the present study, the role of TMPRSS2:ERG in cell death, specifically, apoptosis induced by cisplatin was investigated. The TMPRSS2:ERG fusion gene was demonstrated to serve an important role in the regulation of apoptosis induced by cisplatin, and activating transcription factor 5 (ATF5) was suggested to be involved in this process.

\section{Materials and methods}

Cell culture and treatment. Human embryonic kidney 293 (HEK293) cells and the prostate cancer cell lines DU145 and VCaP cells were purchased from the American Type Culture Collection (Manassas, VA, USA) and the cells were grown in Dulbecco's modified Eagle's medium (Gibco; Thermo Fisher Scientific, Inc., Waltham, MA, USA), supplemented with $10 \%$ fetal bovine serum (Gibco; Thermo Fisher Scientific, Inc.), in a humidified atmosphere containing $5 \% \mathrm{CO}_{2}$ at $37^{\circ} \mathrm{C}$. Cells were plated in 6-well plates and treated at the indicated times or doses with cisplatin (Sigma-Aldrich, St. Louis, MO, USA).

DNA constructs and transfection. cDNA corresponding to the most common truncated ERG ( $\triangle \mathrm{ERG})$, the TMPRSS2:ERG fusion gene (T1/E5), was constructed using pEGP-C1 (Clontech Laboratories, Inc., Mountainview, CA, USA) with PstI and BglII sites to generate a green fluorescent protein (GFP)- $\triangle$ ERG over-expressing vector. Kanamycin resistant colonies were selected and verified by sequencing. Cells were transfected with FugeneHD (Roche Diagnostics, Basel, Switzerland) following the manufacturer's instructions. In brief, $5 \mu$ l FugeneHD was diluted with $50 \mu$ l Opti-MEM (Gibco; Thermo Fisher Scientific, Inc.), mixed and incubated at room temperature for $5 \mathrm{~min}$. Subsequently, $1.5 \mu \mathrm{g}$ plasmid DNA was added to the diluted FugeneHD and mixed, followed by incubation for $20 \mathrm{~min}$ at room temperature, following which the FugeneHD-DNA mixture was added to the cells.

To establish the stable cell line, the HEK293 cells were transfected as described above. Following selection with $0.8 \mathrm{mg} / \mathrm{ml} \mathrm{G} 418$ (Gibco, Thermo Fisher Scientific, Inc.) for 14 days, resistant clones were tested for the expression of $\triangle E R G$. The selected stable cell line was designated as $\Delta 293$, and the cells were cultured further in the medium supplemented with $300 \mu \mathrm{g} / \mathrm{ml} \mathrm{G} 418$ for maintenance. Cells transfected with the GFP-empty vector were designated as E293 and were used as a control.

Scratch assay. Cells were seeded at $1 \times 10^{5}$ cells/well in triplicate in collagen IV coated 6-well plates (Corning Incorporated, Corning, NY, USA). After 24 h, a scratch through the central axis of the plate was gently made using a pipette tip. The migration of $\Delta 293$ and E293 cells into the scratched region was assessed quantitatively at $12 \mathrm{~h}$ following the introduction of the scratch in the monolayer.

Cell proliferation assay. Cells were seeded at $5 \times 10^{3}$ cells/well into a 96-well plate. Cell proliferation was examined using a Cell Counting Kit-8 (Dojindo Molecular Technologies, Inc., Kumamoto, Japan) following the manufacturer's instructions. Absorbance at $450 \mathrm{~nm}$ was measured in quintuplicate using a Tecan Infinite M200 plate reader (Tecan Trading AG, Männedorf, Switzerland) at the indicated time points.

$\triangle E R G$ knock down. Knockdown of $\triangle \mathrm{ERG}$ expression was achieved by the transfection of $\triangle E R G$ short interfering RNA (siRNA). The $\triangle E R G$ siRNA was sequenced by Shanghai GenePharma Co., Ltd., (Shanghai, China), the sequences were as follows: Sense, 5'-CCAGACGUCAACAUCUUGUTT-3' and antisense, 5'-ACAAGAUGUUGACGUCUGGTT-3', which were synthesized by. A nonspecific control strand (UUCUCC GAACGUGUCACGUTT) was used as the negative control (NC).

The siRNA was transfected into the cells using Lipofectamine 2000 (Invitrogen; Thermo Fisher Scientific, Inc.) following the manufacturer's instructions.

Immunoblot analysis. Cells were harvested at the indicated times and washed three times in phosphate-buffered saline (PBS) prior to lysis. Protein concentrations were measured using a Bicinchoninic Acid Protein Assay Reagent kit (Pierce Biotechnology, Inc., Rockford, IL, USA). Equal amounts of the protein $(20 \mu \mathrm{g})$ were subjected to 12 or $15 \%$ sodium dodecyl sulfate polyacrylamide gel electrophoresis (Bio-Rad Laboratories, Inc., Hercules, CA, USA), then transferred to polyvinylidene fluoride membranes (EMD Millipore, Billerica, MA, USA), which were incubated first with the primary antibody at $4{ }^{\circ} \mathrm{C}$ overnight, followed incubation with IR Dye-conjugated secondary antibodies for $1 \mathrm{~h}$ at room temperature. The primary antibodies used were as follows: Rabbit polyclonal anti-ERG (1:500; Santa Cruz Biotechnology, Inc., Dallas, TX, USA; cat. no. sc-376293); rabbit polyclonal anti-ATF5 (1:500; Santa Cruz Biotechnology, Inc.; sc-377168); rabbit monoclonal anti-cleaved-caspase-3 (1:1,000; Cell Signaling Technology, Inc., Danvers, MA, USA; cat. no. 9661); rabbit polyclonal anti-poly ADP ribose polymerase (PARP-1; 1:1,000; Cell Signaling Technology, Inc.; cat. no. 9542); mouse monoclonal anti- $\beta$-actin (1:1,000; Cell Signaling Technology, Inc.; cat. no. 3700); rabbit monoclonal anti-H3 (1:1,000; Cell Signaling Technology, Inc.; cat. no. 4499); and mouse monoclonal anti- $\gamma \mathrm{H} 2 \mathrm{AX}$ (1:3,000; EMD Millipore; cat. no. 05-636). Alexa Fluor 488-conjugated goat anti-mouse (1:5,000; cat. no. A-11001) and goat anti-rabbit (1:5,000; cat. no. A11008) IgG were obtained from Thermo Fisher Scientific, Inc.. The results were observed using an Odyssey Infrared Imaging System (Li-Cor, Inc., Lincoln, NB, USA).

Flow cytometry. Cells were harvested at $48 \mathrm{~h}$ post cisplatin treatment. Cells were centrifuged at $300 \mathrm{x} \mathrm{g}$ for $5 \mathrm{~min}$ at $4^{\circ} \mathrm{C}$ and washed with PBS three times, then resuspended in $1 \mathrm{X}$ Binding Buffer. Subsequently, Annexin V-phycoerythrin 
and 7-aminoactinomycin D (EMD Millipore) were added, and cells were incubated at room temperature for $5 \mathrm{~min}$ in the dark, following which the apoptotic cells were analyzed by flow cytometry (FC500 MCL; Beckman Coulter, Inc., Brea, CA, USA).

Reverse transcription-quantitative polymerase chain reaction $(R T-q P C R)$. Total RNA from E293 and $\Delta 293$ cells was extracted using TRIzol (Invitrogen; Thermo Fisher Scientific, Inc.), and $5 \mu \mathrm{g}$ of total RNA was reverse-transcribed to cDNA using SuperscriptIII Reverse Transcriptase (Invitrogen; Thermo Fisher Scientific, Inc.). Prior to this, the RNA was treated with DNase I (Sangon Biotech Co., Ltd., Shanghai, China) to remove DNA contamination. The $20 \mu \mathrm{l}$ PCR reaction was set up using SYBR Green Premix Ex Taq kit (Takara Bio, Inc., Otsu, Japan) on a 96-well plate, and conducted using a ABI Prism Sequence Detection System 7500 (Applied Biosystems, Inc.; Thermo Fisher Scientific, Inc.) with the following PCR conditions: $95^{\circ} \mathrm{C}$ for $1 \mathrm{~min} ; 95^{\circ} \mathrm{C}$ for $10 \mathrm{sec}$, $60^{\circ} \mathrm{C}$ for $1 \mathrm{~min}$, repeated for 40 cycles. The primers used were as follows: Sense, 5'-GAGTGGGCGGTGAAAGAA TA-3' and antisense, 5'-AGAAGGATGTCGGCGTTGTA-3' for ERG; sense, 5'-TGCGTGACATAAGGAGAA-3' and antisense, 5'-AAGGAAGGCTGGAAGAGT-3' for $\beta$-actin; sense, 5'-TGGAAAGCGTAGACAAGGAGAT-3' and antisense, 5'-AAGGCTCTAGGTGGTCATTCAG-3' for BCL-XL; sense, 5'-CCATCTACTGCCGCAACGAG-3' and antisense, 5'-CTC AGAGCCGCCGAC TTGTT-3' for ATF5; sense, 5'-GGATTG TGGCCTTCTTTGA-3' and antisense, 5'-GTGCCGGTTCAG GTACTCA-3' for BCL-2; sense, 5'-GGACGGCGGTGATGG ACG-3' and antisense, 5'-GAAGCAAAAGGGCCCCTGT-3' for BAX; and sense, 5'-CCGCCACTACCACCACTTGA-3' and antisense, 5'-GGGAACGCAGCGAACCGAAT-3' for BIM. Data analysis was performed using the $2^{-\Delta \Delta C q}$ method for relative quantification (13), and all samples were normalized to $\beta$-actin, which served as an endogenous control.

Chromatin immunoprecipitation (ChIP). ChIP assays were performed using the EZ ChIP kit (EMD Millipore) according to the manufacturer's instructions. Briefly, cross-linked chromatin was sonicated and immunoprecipitated with the indicated antibody. Three independent ChIP analyses were performed to prepare the DNA for future analysis. Immunoprecipitated DNA was purified and quantified by RT-qPCR as described above. The primers were as follows: Sense, 5'-AAAAGACGG ACATCCACCTCG-3' and antisense, 5'-CAAGGGATGGAG AAAAGGACG-3' for ATF5.

Statistical analysis. The data are presented as the mean \pm standard deviation from a minimum of three independent experiments. The differences between groups were assessed using an unpaired two-tailed Student's t-test or one-way analysis of variance. $\mathrm{P}<0.05$ was considered to indicate a statistically significant difference.

\section{Results}

$\triangle E R G$ inhibits cisplatin-induced apoptosis in PCa cell lines. The present study evaluated the toxicity of cisplatin on DU145 and VcaP cells. It was observed that these two types of cell were relatively resistant to the toxic effect of cisplatin as only at concentrations of $50 \mu \mathrm{M}$ or higher was significant cell death observed (data not shown). To examine the function of fusion genes in apoptosis, GFP-tagged TMPRSS2:ERG (T1/E5) $(\triangle E R G)$ was transiently expressed in DU145 cells, which do not express this fusion gene. The cells were then treated with $50 \mu \mathrm{M}$ cisplatin for $48 \mathrm{~h}$, and the apoptotic cell population was analyzed by flow cytometry. As shown in Fig. 1A, the $\triangle \mathrm{ERG}$ protein was expressed in the transfected DU145 cells as indicated by western blotting. Following cisplatin treatment, the apoptotic cell ratio in the control DU145 cells was $30.59 \%$. However, this ratio was reduced to $14.67 \%$ in $\triangle \mathrm{ERG}$ transfected DU145 cells ( $\mathrm{P}<0.01$; Fig. 1B). To further investigate the function of $\triangle E R G$ in apoptosis, VCaP cells, which harbor the TMPRSS2:ERG fusion gene, were transfected with siRNAs to knockdown the expression of the fusion gene (Fig. 1C). Consistent with the idea that the fusion protein inhibits apoptosis, the apoptotic cell ratio increased from $13.59 \%$ in control cells to $21.35 \%$ in siRNA-transfected cells ( $\mathrm{P}<0.05$; Fig. 1D).

Expression of $\triangle E R G$ renders HEK293 cells resistant to cisplatin-induced apoptosis. The toxic effects of cisplatin on HEK 293 cells were also examined. It was demonstrated that compared with PCa cells, HEK293 cells were more sensitive to the toxic effect of cisplatin, as at a concentration of $1 \mu \mathrm{M}$ marked cell death was observed and at $100 \mu \mathrm{M}$, almost all the cells had undergone cell death (data not shown). To determine whether $\triangle \mathrm{ERG}$ only has the anti-apoptosis function in PCa cells but not in other types of cell, a stable cell line expressing the $\triangle E R G$ gene was established using HEK 293 cells ( $\Delta 293$ cells), with cells transfected with the empty plasmid serving as controls (E293 cells). GFP fluorescence demonstrated that the majority of the $\triangle E R G$ fusion protein was localized within the nucleus, and western blot analysis using a commercial anti-ERG antibody confirmed the expression of $\triangle E R G$ (Fig. 2A). The effect of $\triangle E R G$ on cell growth was investigated and the results indicated that the expression of the $\triangle E R G$ gene in HEK293 cells did not affect cell growth (data not shown). To further examine whether $\triangle E R G$ does indeed serve a role in protecting cells from cisplatin-induced apoptosis, $\Delta 293$ cells were treated with $50 \mu \mathrm{m}$ cisplatin for $48 \mathrm{~h}$. The cell viability of E293 cells was $10 \%$ with cisplatin treatment, while in $\Delta 293$ cells, the viability was $\sim 46 \%$, suggesting that $\Delta E R G$ can protect $\Delta 293$ cells from cell death induced by cisplatin treatment (Fig. 2D). Further analysis revealed that the apoptotic cell ratio in E293 cells was $67.26 \%$, while in $\Delta 293$ cells it was $25.89 \%(\mathrm{P}<0.01$; Fig. 2E). To verify that apoptosis had indeed occurred, a number of apoptosis-associated proteins, including caspase 3 and PARP-1, were examined by western blotting following cisplatin treatment. As shown in Fig. 2F, caspase 3 and PARP-1 cleavage was apparent in E293 cells following cisplatin treatment, however, was less abundant in $\Delta 293$ cells under the same conditions. To further demonstrate that $\triangle E R G$ was responsible for the protective effect, $\triangle E R G$ was knocked down in $\Delta 293$ cells using specific siRNA (Fig. 2G), which indicated that loss of $\triangle E R G$ can restore the sensitivity of $\Delta 293$ cells to cisplatin treatment (Fig. $2 \mathrm{H}$ ). Taken together, these data demonstrate that $\triangle \mathrm{ERG}$ is able to protect cells from apoptosis induced by cisplatin. 
A
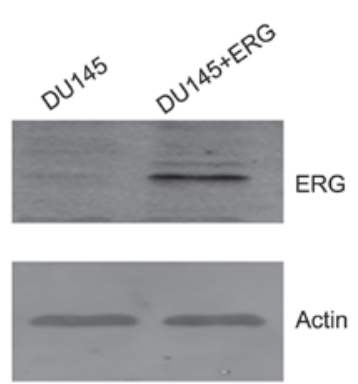

C

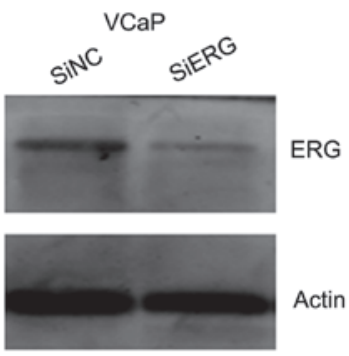

B

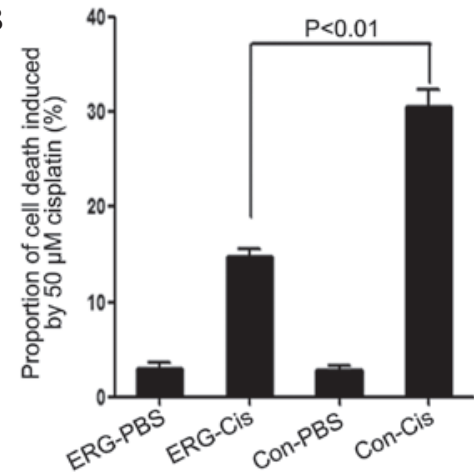

D

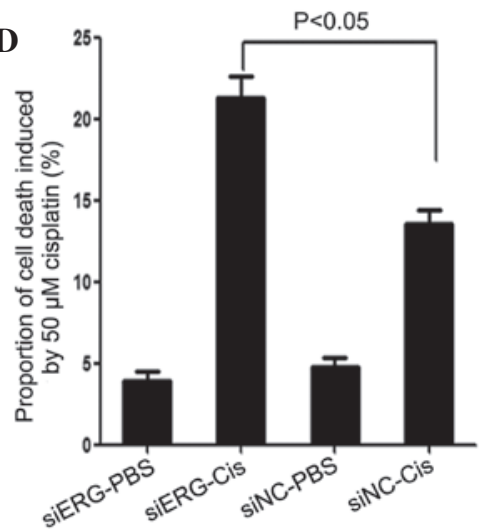

Figure 1. Effects of $\triangle \mathrm{ERG}$ on cisplatin-induced apoptosis in DU145 and VCaP cells. At $24 \mathrm{~h}$ following transfection, cells were treated with $50 \mu \mathrm{M}$ cisplatin for $48 \mathrm{~h}$, and apoptosis was detected by 7-aminoactinomycin D and annexin- $\mathrm{V}$ phycoerythrin double staining. (A) Western blotting of $\triangle$ ERG protein expression in DU145 cells transfected with GFP-vector or GFP- $\Delta$ ERG. (B) Quantitative results of cisplatin-induced apoptosis, as measured by flow cytometry analysis in DU145 cells. (C) Western blotting of $\triangle$ ERG protein expression in VCaP cells transfected with control or specific siRNA directed against $\triangle E R G$. (D) Quantitative results of cisplatin-induced apoptosis as measured by flow cytometry analysis in VCaP cells. $\Delta$ ERG, truncated v-E-twenty-six avian erythroblastosis virus E26 oncogene homolog; GFP, green fluorescent protein; siRNA, short interfering RNA; PBS, phosphate-buffered saline; Cis, cisplatin; siNC, si-negative control; Con, control.

A

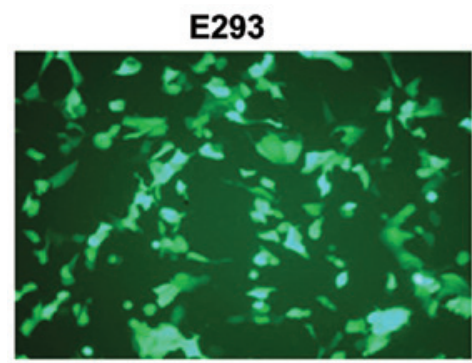

C

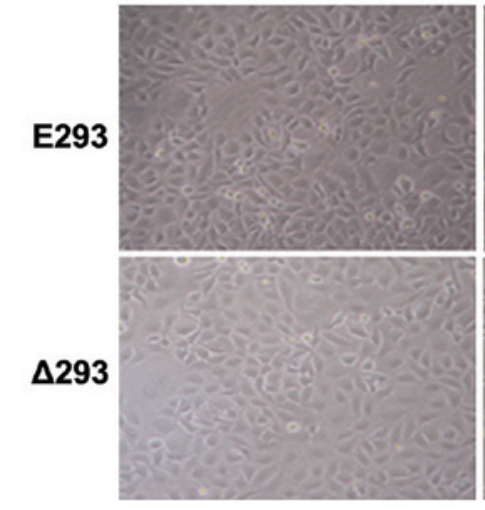

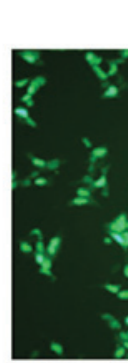

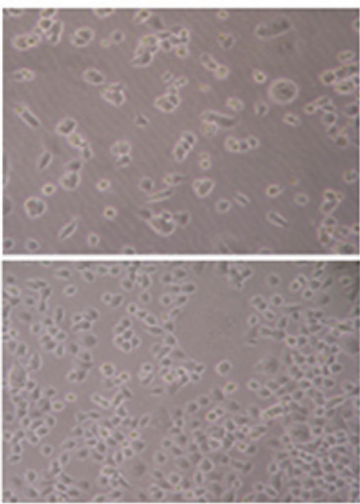

$\Delta 293$
B

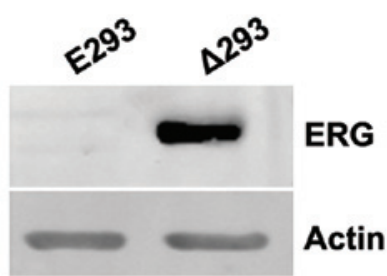

D
$P<0.01$

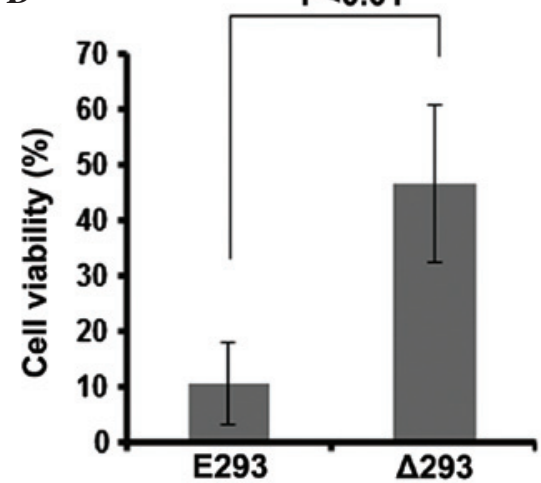

Figure 2. Effects of $\Delta E R G$ overexpression on cisplatin-induced apoptosis in HEK293 cells. (A) Fluorescent images of the expression of $\Delta E R G$ in E293 or $\Delta 293$ cells (magnification, x200). (B) Western blotting for $\Delta$ ERG expression. (C) Representative phase contrast microscopy images of E293 and $\Delta 293$ cell density and morphological alterations following $50 \mu \mathrm{M}$ cisplatin treatment for $48 \mathrm{~h}$ (magnification, $\mathrm{x} 200$ ). (D) Cell viability as measured with a Cell Counting Kit-8 kit. $\triangle E R G$, truncated v-E-twenty-six avian erythroblastosis virus E26 oncogene homolog; E293, p-enhanced green fluorescent vector-transfected HEK293 cells; $\Delta 293, \Delta$ ERG-transfected HEK293 cells. 
$\mathbf{E}$

PBS cisplatin

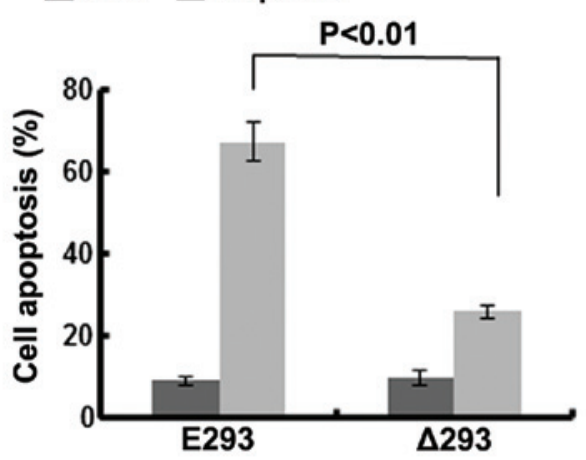

G



F

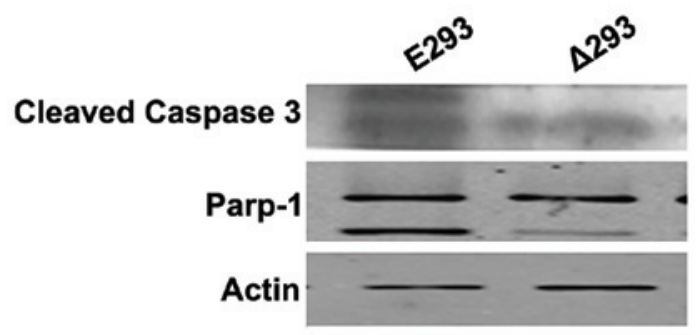

PBS

H

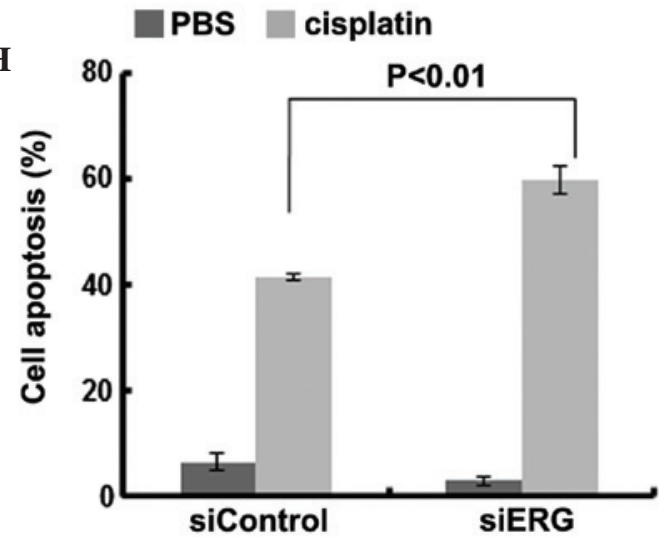

Figure 2. Continued. Effects of $\triangle E R G$ overexpression on cisplatin-induced apoptosis in HEK293 cells. (E) Quantitative results of flow cytometry analyses for cell apoptosis using 7-aminoactinomycin D and annexin-V phycoerythrin double staining. Cells were treated with $50 \mu \mathrm{M}$ cisplatin for $48 \mathrm{~h}$. (F) Western blotting for cleaved caspase-3 and cleaved PARP-1. E293 and $\Delta 293$ cells were treated with $50 \mu \mathrm{M}$ cisplatin for $48 \mathrm{~h}$, total cell lysates were prepared and $10 \mu \mathrm{g}$ aliquots of total proteins were subjected to $15 \%$ sodium dodecyl sulfate-polyacrylamide gel electrophoresis. (G) Western blotting was used to measure $\Delta$ ERG protein expression in $\Delta 293$ cells transfected with control or specific siRNA against $\Delta$ ERG. $(\mathrm{H})$ Quantitative results of flow cytometric analyses for cell apoptosis. $\triangle \mathrm{ERG}$, truncated v-E-twenty-six avian erythroblastosis virus E26 oncogene homolog; E293, p-enhanced green fluorescent vector-transfected HEK293 cells; $\Delta 293, \Delta$ ERG-transfected HEK293 cells; PARP1, poly ADP-ribose polymerase-1; siRNA, short interfering RNA; PBS, phosphate-buffered saline; siNC, si-negative control.
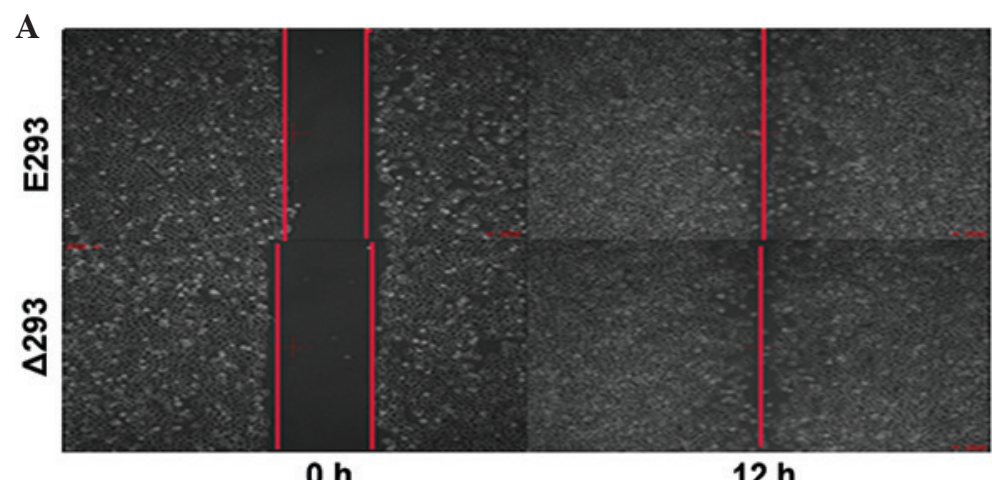

$12 \mathrm{~h}$
B

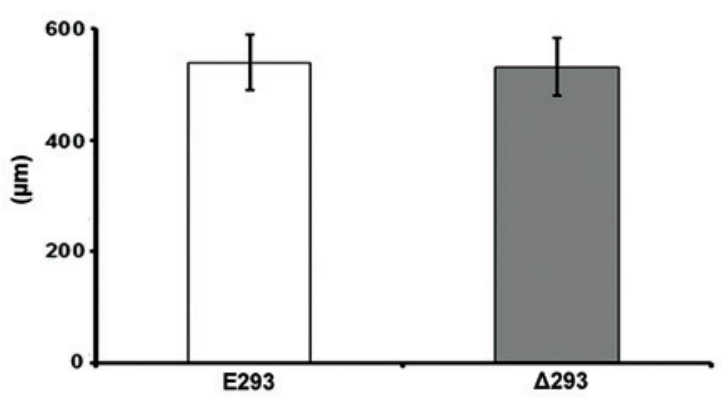

Figure 3. Effects of $\Delta$ ERG overexpression on cell migration. E293 and $\Delta 293$ cells were seeded at $1 \times 10^{5}$ cells/well in triplicate in collagen IV coated 6-well plates. After $24 \mathrm{~h}$, a scratch through the central axis of the plate was gently made using a pipette tip. (A) Representative images of a scratch assay measuring cell migration of E293 and $\Delta 293$ cells. (B) Quantitative results for migration analyses. $\Delta$ ERG, truncated v-E-twenty-six avian erythroblastosis virus E26 oncogene homolog; E293, p-enhanced green fluorescent vector-transfected HEK293 cells; $\Delta 293, \Delta$ ERG transfected HEK293 cells.

It has been previously reported that a major function of TMPRSS2:ERG is to promote PCa cell migration and invasion (1,14-18). Therefore, the effect of $\triangle \mathrm{ERG}$ on cell migration was investigated using a scratch assay. Of note, no significant difference was observed in the scratch assay results between the $\Delta 293$ and E293 cells (Fig. 3), suggesting that overexpressing $\triangle$ ERG did not affect cell motility, at least in HEK293 cells. $\triangle E R G$ upregulates expression of the anti-apoptotic gene ATF5. Considering the potential anti-apoptotic function of $\triangle E R G$, and given that the protein functions as a transcription factor, whether it was able to influence the expression of key regulators in apoptosis, such as members of the BCL-2 family, was of interest. Therefore, RT-qPCR was conducted to examine the expression of the BCL-2 family members BCL-2, BCL-XL, $\mathrm{BIM}$ and $\mathrm{BAX}$ in the presence and absence of $\triangle \mathrm{ERG}$. Notably, 
A

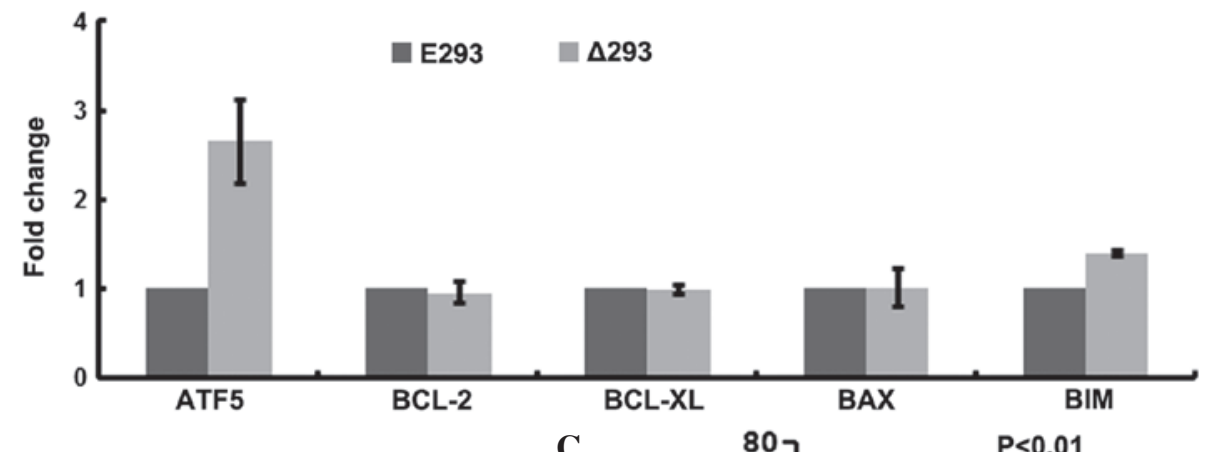

B

C

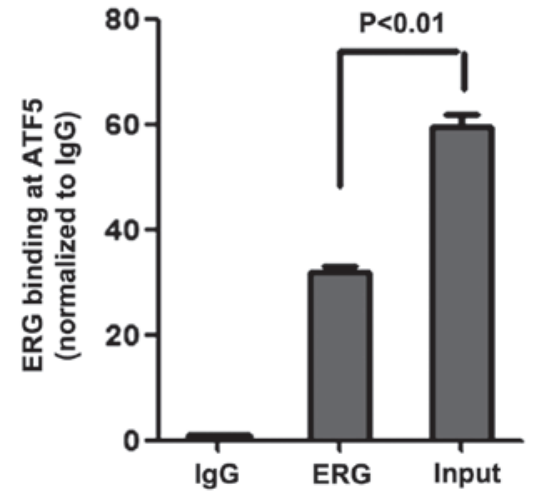

Figure 4. ATF5 expression is upregulated in $\Delta 293$ cells. (A) RT-qPCR detection of apoptosis-associated genes in E293 cells and $\Delta 293$ cells. (B) $\Delta 293$ cells were transfected with control or specific siRNA against $\triangle E R G$, and expression of ATF5 protein was measured by western blotting. (C) Quantitative results of RT-qPCR analyses associated with ChIP assays. ATF5, activating transcription factor 5; $\Delta 293$, truncated v-E-twenty-six avian erythroblastosis virus E26 oncogene homolog-transfected HEK293 cells; RT-qPCR, reverse transcription-quantitative polymerase chain reaction; E293, p-enhanced green fluorescent vector-transfected HEK293 cells; siRNA, short interfering RNA; IgG, immunoglobulin G.

A

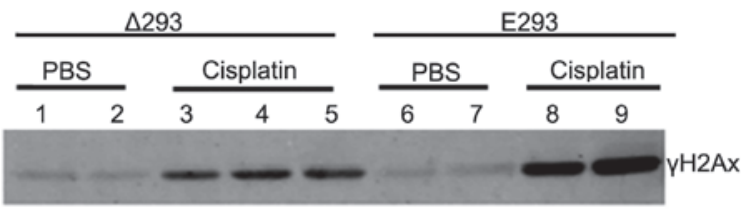

B
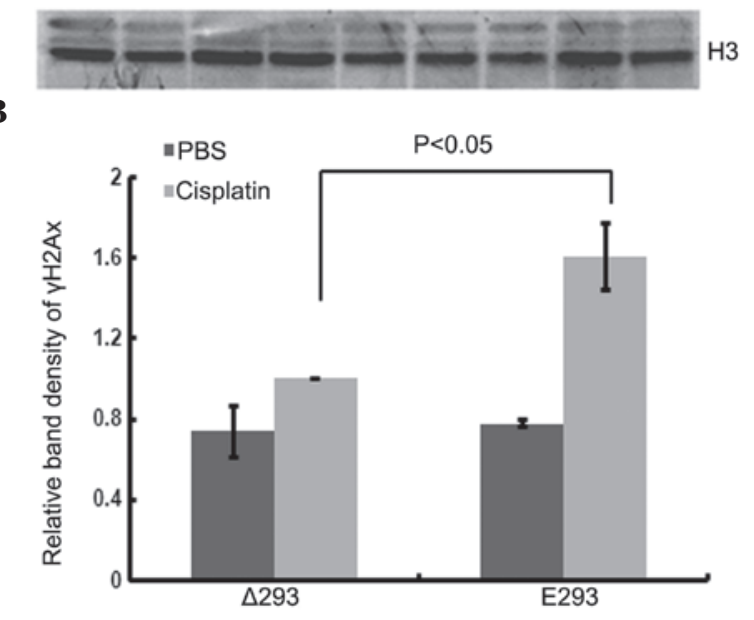

Figure 5. $\triangle$ ERG overexpression reduces cisplatin-induced DNA damage in $\Delta 293$ cells. (A) Western blotting detection of $\gamma \mathrm{H} 2 \mathrm{AX}$ protein in E293 and $\Delta 293$ cells following $50 \mu \mathrm{M}$ cisplatin treatment for $24 \mathrm{~h}$. Lanes 1 and $2, \Delta 293$ cells treated with PBS; lanes 3, 4 and 5, $\Delta 293$ cells treated with $50 \mu \mathrm{M}$ cisplatin; lanes 6 and 7, E293 cells treated with PBS; and lanes 8 and 9, E293 cells treated with $50 \mu \mathrm{M}$ cisplatin. (B) Densitometry analysis of western blotting results. The relative band densities of $\gamma \mathrm{H} 2 \mathrm{AX}$ were normalized to $\mathrm{H} 3$, and the value of the bands from $\Delta 293$ cells treated with cisplatin was arbitrarily set as 1 . The values were derived from at least three independent experiments. $\mathrm{P}<0.05$ vs. $\mathrm{E} 293$ is indicated. $\triangle \mathrm{ERG}$, truncated v-E-twenty-six avian erythroblastosis virus E26 oncogene homolog; $\Delta 293, \Delta$ ERG-transfected HEK293 cells; E293, p-enhanced green fluorescent vector-transfected HEK293 cells; PBS, phosphate-buffered saline; H3, histone H3. none of these genes showed differential expression between E293 and $\triangle 293$ cells (Fig. 4A). However, ATF5 (also known as ATFx), a member of the basic zipper protein ATF/cAMP response element-binding protein family which can suppress apoptosis (19), was observed to be up-regulated in $\Delta 293$ cells (Fig. 4A). To further examine the possibility that ATF5 expression was regulated by $\triangle E R G$, the protein levels of ATF5 were examined by western blotting in $\Delta 293$ cells, transfected with either control siRNA or with siRNA designed to knockdown $\triangle E R G$. As shown in Fig. 4B, the ATF5 protein levels were reduced when $\triangle E R G$ was knocked down, suggesting that $\triangle E R G$ serves a role in the regulation of ATF5 expression. As ERG is a transcription factor, it was then investigated whether $\triangle E R G$ is able to bind directly to the ATF5 gene to regulate ATF5 expression. To investigate this, a ChIP assay was performed in $\Delta 293$ cells using a specific anti-ERG antibody, which indicated that $\triangle E R G$ was able to bind to the ATF5 promoter region in $\Delta 293$ cells (Fig. 4C).

Expression of $\triangle E R G$ protects cells from cisplatin-induced DNA damage. As a classical DNA damage agent, cisplatin is thought to induce apoptosis, at least in part, through the generation of DNA damage. Therefore, the effect of $\triangle E R G$ expression on cisplatin-induced DNA damage was assessed. E293 and $\Delta 293$ cells were treated with cisplatin $(50 \mu \mathrm{M})$ for $24 \mathrm{~h}$, and the level of $\gamma \mathrm{H} 2 \mathrm{AX}$, a sensitive indicator for DNA damage, was examined by western blotting. As shown in Fig. 5, cisplatin treatment increased the level of $\gamma \mathrm{H} 2 \mathrm{AX}$ in the $\Delta 293$ and E293 cells. However, the increase was much smaller in the $\Delta 293$ cells (lanes 3, 4 and 5) compared with the E293 cells (lanes 8 and 9), indicating that the expression 
of $\triangle E R G$ may partially protect cells from cisplatin-induced DNA damage.

\section{Discussion}

The potential role of TMPRSS2:ERG fusion in prostate tumorigenesis and tumor progression has been under intensive investigation. Enhancing cell invasion and metastasis has been regarded as the major function of this protein, and although overexpression of ERG was observed to induce a neoplastic phenotype in prostate cells in certain studies $(5,6)$, it was thought that the protein may require the cooperation of additional genes, such as phosphatase and tensin homolog or members of the phosphatidylinositol 3-kinase pathway $(14,15)$, to result in transformation. However, its association with apoptosis/cell death, a major mechanism for the majority of cancer chemotherapeutic drugs, was relatively unexplored prior to the current study. Platinum chemotherapy drugs, such as cisplatin and carboplatin, have moderate single-agent activity in PCa (12). However, when combined with a taxane and estramustine, high response rates were observed in several clinical trials $(20,21)$. Considering the high prevalence of TMPRSS2:ERG fusion in patients with $\mathrm{PCa}$, its potential role in the relatively low response rate of PCa to cisplatin was explored.

Indeed, PCa cells were observed to be relatively resistant to the cytotoxic effects of cisplatin. For example, only at doses of $50 \mu \mathrm{M}$ or above were significant levels of cell death induced by cisplatin in either DU145 or VCaP cells (data not shown). By contrast, significant cell death was not induced in HEK293 cells by cisplatin at concentrations as low as $1 \mu \mathrm{M}$, and high levels of cell death was observed at $100 \mu \mathrm{M}$ (data not shown). Therefore, $50 \mu \mathrm{M}$ was selected as the test concentration for all subsequent experiments. The response of the PCa cell lines to cisplatin treatment was investigated in DU145 cells, which do not express the fusion gene and $\mathrm{VCaP}$ cells, which harbor the fusion gene. This indicated that cisplatin is able to induce up to $30 \%$ apoptosis in DU145 cells, while the drug induces $\sim 13 \%$ apoptosis in VCaP cells (Fig. 1). However, when $\triangle$ ERG was expressed in DU145 cells, the apoptotic cell ratio was reduced to $\sim 15 \%$. In comparison, knocking down the endogenous fusion gene expressed in the VCaP cell line led to an increased apoptotic cell ratio. Thus, this indicates that $\triangle E R G$ may influence the response of these cells to cisplatin. To discover whether or not the anti-apoptotic effect of $\triangle E R G$ was limited to PCa cells, $\triangle E R G$ was introduced into the HEK293 cells. Notably, no significant effect of $\triangle$ ERG on HEK293 cell growth or migration was observed (data not shown and Fig. 3). These observations are in contradiction to the report by Tian et al (7) in which it was shown that the overexpression of TMPRSS2:ERG in PCa cells markedly increased invasion. A possible explanation may be that different types of cells were used, with HEK293 cells originating from the kidney rather than the prostate, and thus may have a different genetic background. Alternatively, an effect of $\triangle \mathrm{ERG}$ on HEK293 cells was observed with respect to apoptosis. Although cisplatin induced apoptosis in $70 \%$ of the HEK 293 cells, which only expressed the GFP-tag, the apoptotic cell ratio was around $30 \%$ in the $\Delta 293$ cells that expressed the $\Delta$ ERG (Fig. 2). An additional piece of evidence for the anti-apoptotic function of $\triangle \mathrm{ERG}$ comes from the observation that knocking down $\triangle \mathrm{ERG}$ in $\Delta 293$ cells rendered the cells sensitive to cisplatin (Fig. 2). Taken together, these data indicate an anti-apoptotic effect of $\triangle \mathrm{ERG}$. Therefore, future treatment planning for patients with PCa may be enhanced by assessing the status of the TMPRSS2 gene fusion.

Once the anti-apoptotic function of the fusion gene had been determined in the current study, the underlying molecular mechanism was further probed. The BCL-2 family of proteins include both anti-apoptotic (BCL-2, BCL-XL and MCL-1) and apoptotic (BAK, BAX, BID and BIM) members (22). The regulation and balance of the BCL-2 family proteins in a particular cell results in either the inhibition or induction of the apoptotic signaling pathways (23). Therefore, the expression of several BCL-2 family members was examined. Notably, none of the BCL-2 family members examined exhibited differential expression between the E293 and $\Delta 293$ cells (Fig. 4), suggesting that they may not be involved in the anti-apoptotic activity of $\triangle E R G$. However, it was observed that the expression of ATF5, a transcriptional activator with anti-apoptotic activity, was increased in $\Delta 293$ cells. It has been reported that when ATF5 was stably expressed in an IL-3-dependent cell line, apoptosis was suppressed through cytokine deprivation (24). Additionally, overexpression of ATF5 suppressed apoptosis induced by serum withdrawal in HeLa cells (25). By contrast, inhibition of endogenous ATF5 activity by the introduction of a dominant-negative form of ATF5 led to apoptosis in asynchronously growing cells $(24,25)$. To determine whether $\triangle \mathrm{ERG}$ was involved in the regulation of ATF5 expression, $\triangle E R G$ was knocked down in $\Delta 293$ cells, and the protein levels of ATF5 were examined by western blotting. As shown in Fig. 4, knock down $\triangle$ ERG resulted in a reduction in ATF5 expression. Subsequently, ChIP analysis was performed, and the results indicated that $\triangle \mathrm{ERG}$ is able to bind directly to the promoter region of ATF5 (Fig. 4). Based on these observations, $\triangle E R G$ is proposed to potentially upregulate the expression of ATF5, which in turn exerts anti-apoptotic activities through the activation of downstream signaling pathways.

Finally, since cisplatin-induced DNA damage is considered to be a major factor in triggering apoptosis, it was examined whether $\triangle E R G$ is able to influence cisplatin-induced DNA damage. $\gamma \mathrm{H} 2 \mathrm{AX}$ is regarded to be a sensitive indicator for DNA damage (26). The results of the present study indicated that expression of the $\gamma \mathrm{H} 2 \mathrm{AX}$ protein was significantly reduced in $\Delta 293$ cells, suggesting that overexpression of $\triangle E R G$ may protect cells from cisplatin-induced DNA damage (Fig. 5). The role of TMPRSS2:ERG in the DNA damage process remains controversial. TMPRSS2:ERG has been previously shown to interact with the enzyme PARP1 and the catalytic subunit of DNA protein kinase in a DNA-dependent manner, and overexpression of similar fusion genes induces DNA double-strand breaks (27). In addition, Han et al (28) observed that overexpression of TMPRSS2:ERG increases PARP1 activity and clonogenic survival (28). Haffner et al (29) reported that androgen signaling promotes co-recruitment of the androgen receptor and topoisomerase II beta (TOP2B) to sites of TMPRSS2:ERG genomic breakpoints, triggering recombinogenic TOP2B-mediated DNA double-strand breaks (DSBs). Furthermore, androgen stimulation resulted in de novo production of TMPRSS2:ERG fusion transcripts in a process that required TOP2B and components of 
the DSB repair machinery (29). Thus, how $\triangle \mathrm{ERG}$ may interfere with the DNA damage process remains clear, and the potential link between $\triangle E R G$ and DNA damage is of great interest for future studies.

In summary, the current study indicated that the $\Delta \mathrm{ERG}$ protein is able to inhibit cisplatin-induced DNA damage and cell death, and that the ATF5 gene may participate in such events.

\section{Acknowledgements}

The current study was supported by grants from the National Natural Science Foundation of China (grant nos. 81172692, 81302398, 81502752 and 81373036), Department of Science and Technology, Zhejiang Province (grant no. 2013C14016), the Hangzhou City S\&T Committee (grant no. 20130633B50) and the Zhejiang Provincial Natural Science Foundation (grant no. Q16H220001).

\section{References}

1. Siegel R, Naishadham D and Jemal A: Cancer statistics for Hispanics/Latinos, 2012. CA Cancer J Clin 62: 283-298, 2012.

2. Tomlins SA, Rhodes DR, Perner S, Dhanasekaran SM, Mehra R, Sun XW, Varambally S, Cao X, Tchinda J, Kuefer R, et al: Recurrent fusion of TMPRSS2 and ETS transcription factor genes in prostate cancer. Science 310: 644-648, 2005.

3. Falzarano SM and Magi-Galluzzi C: ERG protein expression as a biomarker of prostate cancer. Biomark Med 7: 851-865, 2013.

4. Helgeson BE, Tomlins SA, Shah N, Laxman B, Cao Q, Prensner JR, Cao X, Singla N, Montie JE, Varambally S, et al: Characterization of TMPRSS2:ETV5 and SLC45A3:ETV5 gene fusions in prostate cancer. Cancer Res 68: 73-80, 2008.

5. Tomlins SA, Laxman B, Varambally S, Cao X, Yu J, Helgeson BE, Cao Q, Prensner JR, Rubin MA, Shah RB, et al: Role of the TMPRSS2-ERG gene fusion in prostate cancer. Neoplasia 10: $177-188,2008$

6. Klezovitch O, Risk M, Coleman I, Lucas JM, Null M, True LD, Nelson PS and Vasioukhin V: A causal role for ERG in neoplastic transformation of prostate epithelium. Proc Natl Acad Sci USA 105: 2105-2110, 2008.

7. Tian TV, Tomavo N, Huot L, Flourens A, Bonnelye E, Flajollet S, Hot D, Leroy X, de Launoit Y and Duterque-Coquillaud $\mathrm{M}$ : Identification of novel TMPRSS2:ERG mechanisms in prostate cancer metastasis: Involvement of MMP9 and PLXNA2. Oncogene 33: 2204-2214, 2014.

8. Shao L, Zhou Z, Cai Y, Castro P, Dakhov O, Shi P, Bai Y, Ji H, Shen W and Wang J: Celastrol suppresses tumor cell growth through targeting an AR-ERG-NF-kB pathway in TMPRSS2/ERG fusion gene expressing prostate cancer. PLoS One 8: e58391, 2013.

9. Loehrer PJ and Einhorn LH: Drugs five years later. Cisplatin. Ann Intern Med 100: 704-713, 1984

10. Prestayko AW, D'Aoust JC, Issell BF and Crooke ST: Cisplatin (cis-diamminedichloroplatinum II). Cancer Treat Rev 6: 17-39, 1979.

11. Eastman A: The formation, isolation and characterization of DNA adducts produced by anticancer platinum complexes. Pharmacol Ther 34: 155-166, 1987.

12. Oh WK, Tay MH and Huang J: Is there a role for platinum chemotherapy in the treatment of patients with hormone-refractory prostate cancer? Cancer 109: 477-486, 2007.
13. Livak KJ and Schmittgen TD: Analysis of relative gene expression data using real-time quantitative PCR and the 2(-Delta Delta C(T)) method. Methods 25: 402-408, 2001.

14. Carver BS, Tran J, Gopalan A, Chen Z, Shaikh S, Carracedo A, Alimonti A, Nardella C, Varmeh S, Scardino PT, et al: Aberrant ERG expression cooperates with loss of PTEN to promote cancer progression in the prostate. Nat Genet 41: 619-624, 2009.

15. King JC, Xu J, Wongvipat J, Hieronymus H, Carver BS Leung DH, Taylor BS, Sander C, Cardiff RD, Couto SS, et al: Cooperativity of TMPRSS2-ERG with PI3-kinase pathway activation in prostate oncogenesis. Nat Genet 41: 524-526, 2009.

16. Esgueva R, Perner S, J LaFargue C, Scheble V, Stephan C, Lein M, Fritzsche FR, Dietel M, Kristiansen G and Rubin MA: Prevalence of TMPRSS2-ERG and SLC45A3-ERG gene fusions in a large prostatectomy cohort. Mod Pathol 23: 539-546, 2010.

17. Galluzzi L, Aaronson SA, Abrams J, Alnemri ES, Andrews DW, Baehrecke EH, Bazan NG, Blagosklonny MV, Blomgren K, Borner C, et al: Guidelines for the use and interpretation of assays for monitoring cell death in higher eukaryotes. Cell Death Differ 16: 1093-1107, 2009.

18. Galluzzi L, Vitale I, Abrams JM, Alnemri ES, Baehrecke EH, Blagosklonny MV, Dawson TM, Dawson VL, El-Deiry WS, Fulda S, et al: Molecular definitions of cell death subroutines: Recommendations of the nomenclature committee on cell death 2012. Cell Death Differ 19: 107-120, 2012.

19. Hai TW, Liu F, Coukos WJ and Green MR: Transcription factor ATF cDNA clones: An extensive family of leucine zipper proteins able to selectively form DNA-binding heterodimers. Genes Dev 3: 2083-2090, 1989.

20. Papandreou CN, Daliani DD, Thall PF, Tu SM, Wang X, Reyes A, Troncoso P and Logothetis CJ: Results of a phase II study with doxorubicin, etoposide, and cisplatin in patients with fully characterized small-cell carcinoma of the prostate. J Clin Oncol 20: 3072-3080, 2002.

21. Berry W, Friedland D, Fleagle J, Jackson D, Ilegbodu D, Boehm KA and Asmar L: A phase II study of weekly paclitaxel/estramustine/carboplatin in hormone-refractory prostate cancer. Clin Genitourin Cancer 5: 131-137, 2006.

22. Chipuk JE, Moldoveanu T, Llambi F, Parsons MJ and Green DR: The BCL-2 family reunion. Mol Cell 37: 299-310, 2010.

23. Harris MH and Thompson CB: The role of the Bcl-2 family in the regulation of outer mitochondrial membrane permeability. Cell Death Differ 7: 1182-1191, 2000.

24. Persengiev SP and Green MR: The role of ATF/CREB family members in cell growth, survival and apoptosis. Apoptosis 8: 225-228, 2003.

25. Persengiev SP, Devireddy LR and Green MR: Inhibition of apoptosis by ATFx: A novel role for a member of the ATF/CREB family of mammalian bZIP transcription factors. Genes Dev 16: 1806-1814, 2002

26. Zhou C, Li Z, Diao H, Yu Y, Zhu W, Dai Y, Chen FF and Yang J: DNA damage evaluated by gammaH2AX foci formation by a selective group of chemical/physical stressors. Mutat Res 604: 8-18, 2006.

27. Brenner JC, Ateeq B, Li Y, Yocum AK, Cao Q, Asangani IA, Patel S, Wang X, Liang $\mathrm{H}$, Yu J, et al: Mechanistic rationale for inhibition of poly (ADP-ribose) polymerase in ETS gene fusion-positive prostate cancer. Cancer Cell 19: 664-678, 2011.

28. Han S, Brenner JC, Sabolch A, Jackson W, Speers C, Wilder-Romans K, Knudsen KE, Lawrence TS, Chinnaiyan AM and Feng FY: Targeted radiosensitization of ETS fusion-positive prostate cancer through PARP1 inhibition. Neoplasia 15: 1207-1217, 2013.

29. Haffner MC, Aryee MJ, Toubaji A, Esopi DM, Albadine R, GurelB,Isaacs WB, Bova GS,Liu W, Xu J, etal: Androgen-induced TOP2B-mediated double-strand breaks and prostate cancer gene rearrangements. Nat Genet 42: 668-675, 2010. 